\title{
Comparison of MBE Growth of InSb on Si (001) and GaAs (001)
}

\author{
T. LIEN TRAN,${ }^{1,3}$ FARIBA HATAMI, ${ }^{1} \mathrm{~W}$. TED MASSELINK, ${ }^{1}$ \\ VAS P. KUNETS, ${ }^{2}$ and G.J. SALAMO ${ }^{2}$
}

1.-Department of Physics, Humboldt-Universität zu Berlin, Newtonstr. 15, 12489 Berlin, Germany. 2.-Department of Physics, University of Arkansas, Fayetteville, USA. 3.-e-mail: lien.tran@physik.hu-berlin.de

\begin{abstract}
We describe the epitaxial growth of InSb films on both $\mathrm{Si}$ (001) and GaAs (100) substrates using molecular-beam epitaxy and discuss the structural and electrical properties of the resulting films. The complete $2 \mu \mathrm{m}$ InSb films on GaAs (001) were grown at temperatures between $340^{\circ} \mathrm{C}$ and $420^{\circ} \mathrm{C}$ and with an Sb/In flux ratio of approximately 5 and a growth rate of $0.2 \mathrm{~nm} / \mathrm{s}$. The films were characterized in terms of background electron concentration, mobility, and x-ray rocking curve width. Our best results were for a growth temperature of $350^{\circ} \mathrm{C}$, resulting in room-temperature mobility of $41,000 \mathrm{~cm}^{2} / \mathrm{V} \mathrm{s}$. For the growth of InSb on $\mathrm{Si}$, vicinal $\mathrm{Si}(001)$ substrates offcut by $4^{\circ}$ toward $(110)$ were used. We investigated growth temperatures between $340^{\circ} \mathrm{C}$ and $430^{\circ} \mathrm{C}$ for growth on $\mathrm{Si}(001)$. In contrast to growth on $\mathrm{GaAs}$, the best results were achieved at the high end of the range of $T_{\mathrm{S}}=\mathrm{C}$, resulting in a mobility of $26,100 \mathrm{~cm}^{2} / \mathrm{V}$ s for a $2 \mu \mathrm{m}$ film. We also studied the growth and properties of InSb:Mn films on GaAs with Mn content below 1\%. Our results showed the presence of ferromagnetic ordering in the samples, opening a new direction in the diluted magnetic semiconductors.
\end{abstract}

Key words: InSb, GaAs, Si substrates, electron mobility, x-ray diffraction, $(\mathrm{In}, \mathrm{Mn}) \mathrm{Sb}$, diluted magnetic semiconductor

\section{INTRODUCTION}

The replacement of native oxides with deposited oxides in complementary metal-oxide semiconductor (CMOS) technology opens the door to replacing the Si with semiconductors without high-quality native oxides. For example, the use of InSb in logic applications could allow much lower operating voltages and power dissipation due to the InSb channels reaching saturation at significantly lower electric fields. Epitaxy of InSb onto Si could be done directly or by using an intermediate layer such as GaP, GaAs, or InP. Furthermore, InSb, the narrowest bandgap traditional III-V material, has potential applications in high-speed devices, infrared detectors and magnetic sensors. For these applications, InSb thin films should be grown on semi-insulating

(Received November 30, 2007; accepted September 2, 2008; published online October 7, 2008) infrared transparent substrates. Although there is a large lattice mismatch (14.6\%) with InSb, GaAs is still an attractive substrate material, due to its chemical stability and high resistivity. The epitaxial growth of InSb on GaAs substrates has been demonstrated by several groups using a variety of preparation methods, such as molecular beam epitaxy, ${ }^{1,2}$ liquid phase epitaxy ${ }^{3}$ and metalorganic chemical vapor deposition (MOCVD). ${ }^{4}$ From those investigations, it was found that the growth mechanism is quite complicated. It depends not only on the substrate orientation and the surface preparation but also strongly on the growth conditions. Fewer papers have been concerned with the heteroepitaxy of InSb on $\mathrm{Si}$ than with the growth on GaAs. 5, 6 The difficulties involved in growth of highquality InSb films on $\mathrm{Si}$ include the large lattice mismatch (19\%), the different thermal expansion coefficients $\left(\alpha_{\mathrm{InSb}} \approx 2 \alpha_{\mathrm{Si}}\right.$ at room temperature $)$ and antiphase domain formation due to the growth of a 
polar semiconductor on a nonpolar semiconductor. ${ }^{7}$ Some problems have been eliminated by the use of tilted substrates, ${ }^{8}$ indium pre-deposition and the insertion of various buffer layers. ${ }^{9-11}$

In our paper we describe, in detail, the growth of InSb on both GaAs (001) and $\mathrm{Si}$ (001) using molecular-beam epitaxy (MBE), and we compare the growth and properties of the resulting InSb films. Specific aspects of our techniques include the incorporation of a low-temperature InSb buffer (for the GaAs substrate), introduction of a GaSb/AlSb superlattice buffer, and the use of a misoriented substrate (for the Si substrate) to restrain the formation of dislocations generated by the lattice mismatch. The study of InSb/GaAs and InSb/Si layers also involved structural characterization by x-ray diffraction and electrical properties by Hall effect measurement. We also describe the growth of (In,Mn)Sb films on GaAs. The magnetotransport and magnetic properties of the films were studied using Hall and magnetization measurements. We present our results of this new III-Mn-V diluted magnetic semiconductor material.

\section{EXPERIMENTAL}

The growth of InSb/GaAs was carried out in a Riber Compact 21T gas-source MBE system equipped with reflection high-energy electron diffraction (RHEED). The semi-insulating (001) GaAs substrates with $10^{7} \Omega \mathrm{cm}$ resistivity at $22^{\circ} \mathrm{C}$ were first etched by standard wafer cleaning recipes before being loaded in to the MBE system. In the MBE system the indium beam is provided by conventional effusion cell and the antimony cell is cracked with temperatures between $800^{\circ} \mathrm{C}$ and $900^{\circ} \mathrm{C}$. Immediately prior to growth, the thin oxide layer on the substrate surface was desorbed at $600^{\circ} \mathrm{C}$ under ultra-high vacuum in a growth chamber, with antimony flux. Then, the substrate temperature was decreased to approximately $300^{\circ} \mathrm{C}$ to grow a $600 \AA \mathrm{InSb}$ layer. Subsequent to the low-temperature growth of this initial InSb layer, the substrate temperature was raised to the intended temperature for growth of $2 \mu \mathrm{m}$ InSb films. Four samples were grown under the same $\mathrm{Sb} / \mathrm{In}$ flux ratio and growth rate, but at various growth temperatures of $340^{\circ} \mathrm{C}, 350^{\circ} \mathrm{C}, 360^{\circ} \mathrm{C}$ and $370^{\circ} \mathrm{C}$. After the growth of the InSb films, the temperature was reduced to approximately $200^{\circ} \mathrm{C}$ under the antimony flux.

The growth of InSb on $\mathrm{Si}(001)$ was also investigated. The Si substrates ( $p$-type, with $50-80 \Omega \mathrm{cm}$ resistivity) $4^{\circ}$ offcut (001) toward (110) were first cleaned in hot $\mathrm{H}_{2} \mathrm{O} \cdot \mathrm{NH}_{4} \mathrm{OH} \cdot \mathrm{H}_{2} \mathrm{O}_{2}$ (4:1:1) and then subjected to fresh $\mathrm{HF}(49 \%)$ and removal in $\mathrm{H}_{2} \mathrm{O}: \mathrm{HCl}: \mathrm{H}_{2} \mathrm{O}_{2} \quad(4: 1: 1)$ and dilute $\mathrm{H}_{2} \mathrm{O}: \mathrm{HF}$ (10:1) solutions before being loaded into the MBE system. We had reduced the substrate temperature to $T_{\mathrm{S}}=$ $1015^{\circ} \mathrm{C}$ to obtain a surface characterized by doubleheight steps. ${ }^{12}$ After the substrate temperature had been cooled to the InSb growth temperature, a 30-period strained-layer superlattice consisting of $10 \AA \mathrm{GaSb} / 10 \AA \mathrm{AlSb}$ periods was incorporated between the substrate and the $2 \mu \mathrm{m}$ InSb layer. We investigated growth temperatures between $340^{\circ} \mathrm{C}$ and $430^{\circ} \mathrm{C}$.

The (In,Mn)Sb films were grown on GaAs substrates under the same growth conditions as for InSb on GaAs, described above. The $\mathrm{Sb} / \mathrm{In}$ flux ratio was approximately 5 , and the growth rate was $2 \AA / \mathrm{s}$. Growth of a $1 \mu \mathrm{m}$ undoped InSb buffer layer was followed by epitaxy of $1 \mu \mathrm{m}$ (In,Mn)Sb film at $T_{\mathrm{Mn}}=$ $670^{\circ} \mathrm{C}$. We estimate the $\mathrm{Mn}$ content to be approximately $0.5 \%$ from the $\mathrm{Mn} / \mathrm{In}$ flux ratio, and confirmed this by high intensity x-ray measurements.

Rocking curves were measured by high-resolution x-ray diffraction (XRD), carried out in a Bede scientific QC1a diffractometer. ${ }^{13}$ Samples were mounted horizontally, with no adhesives, on detachable sample cassettes. The measurements were made in the $\theta-2 \theta$ configuration.

Hall mobility and carrier concentration have been studied using the van der Pauw method ${ }^{14,15}$ on $4 \times 4 \mathrm{~mm}^{2}$ samples. Indium contacts with small dimensions in comparison with the length of an edge were made at corners of the sample by annealing at $200^{\circ} \mathrm{C}$ in a $\mathrm{H}_{2}(20 \%) / \mathrm{N}_{2}(80 \%)$ atmosphere for $2 \mathrm{~min}$. Hall measurements at the magnetic field of $0.5 \mathrm{~T}$ were carried out for temperatures ranging from $9 \mathrm{~K}$ to $300 \mathrm{~K}$ in a closed-cycle helium cryostat.

We used magnetization measurements, done with a superconducting quantum interference device (SQUID), to study the magnetic properties of the (In,Mn)Sb samples with a temperature control system in the range of $2-400 \mathrm{~K}$ and a magnet control system from zero to positive and negative $7 \mathrm{~T}$. Those samples were also examined by Hall measurements for magnetotransport properties on photolithographically patterned Hall bars with a width of $200 \mu \mathrm{m}$ and a length of $1.3 \mathrm{~mm}$. The measurements were carried out with a $7 \mathrm{~T}$ superconducting magnet with a variable temperature insert and a He cryostat.

\section{RESULTS AND DISCUSSION}

The crystal quality of the InSb films can be obtained from the x-ray diffraction measurements. Figure 1a shows the (004) double-crystal x-ray (DCXR) patterns of InSb/GaAs films grown at various growth temperatures. In all patterns, only peaks due to InSb and GaAs were observed. The InSb films were strongly oriented in the (001) direction on the substrate, and the InSb (004) peaks appeared with quite strong intensity. For the sample grown at $350^{\circ} \mathrm{C}$, the InSb (004) peak exhibited the highest intensity, and the full-width at halfmaximum (FWHM) decreased to approximately 380 arcseconds compared to 439, 494, and 549 arcseconds at $340^{\circ} \mathrm{C}, 360^{\circ} \mathrm{C}$, and $370^{\circ} \mathrm{C}$, respectively. These DCXR results imply that the substrate temperature around $350^{\circ} \mathrm{C}$ is suitable to grow $\mathrm{InSb} /$ GaAs with good crystal quality. 

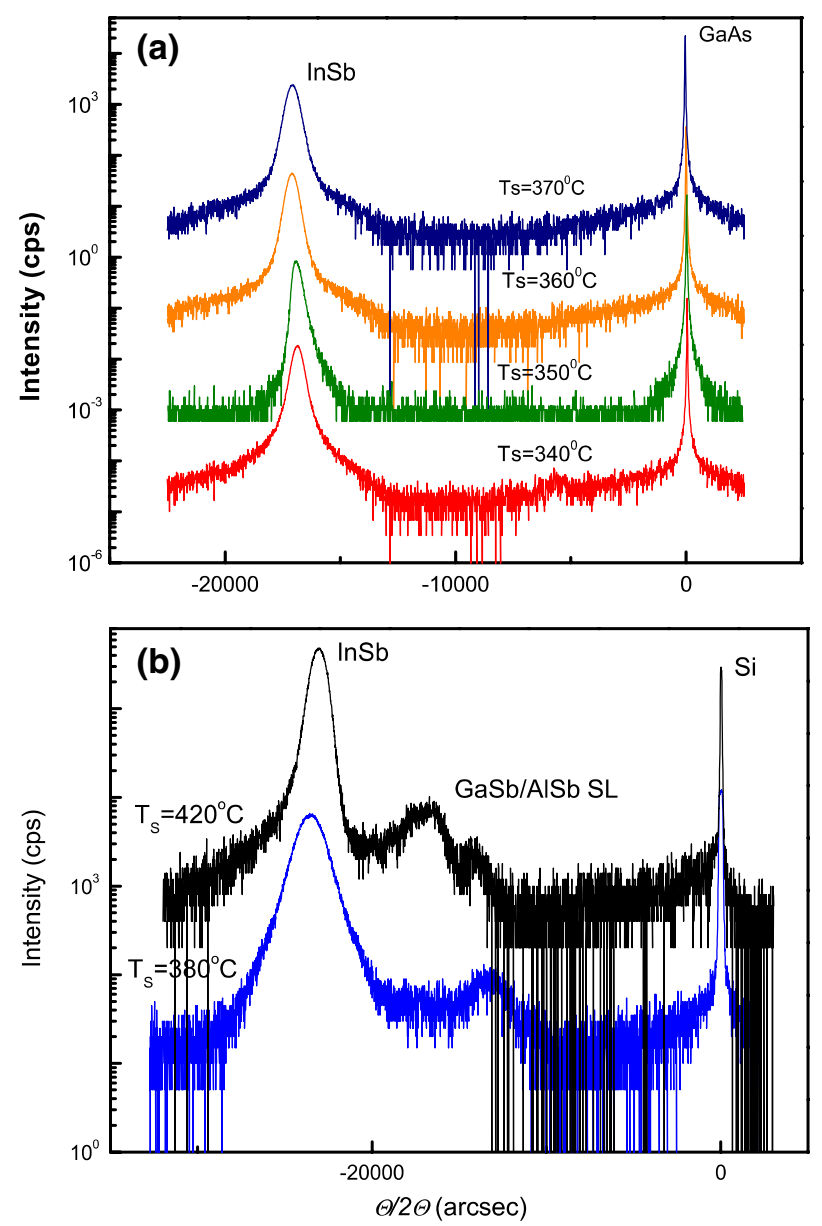

Fig. 1. Double-crystal $x$-ray rocking curves for (a) InSb/GaAs grown at $370^{\circ} \mathrm{C}, 360^{\circ} \mathrm{C}, 350^{\circ} \mathrm{C}$, and $340^{\circ} \mathrm{C}$, and (b) InSb/Si grown at $420^{\circ} \mathrm{C}$ and $380^{\circ} \mathrm{C}$.

In addition, the values of lattice strain were calculated to be smaller than $0.05 \%$, indicating that the films were all nearly $100 \%$ relaxed. This result can be easily understood, because the lattice strain energy builds up to a point where it becomes energetically favorable to form misfit dislocations, when the film is thick enough. At this point, the initially strained film would ideally decompose to a relaxed structure where the generated dislocations relieve a portion of the misfit. The large mismatch between the InSb film- and GaAs substrate-introduced misfit and threading dislocations has been investigated and reported by Weng et al. ${ }^{1,16}$

In the case of $\mathrm{InSb} / \mathrm{Si}$, it is much more difficult to obtain a good crystal quality of InSb film with a smooth surface. To improve the crystal quality, we applied several techniques. One of the techniques was the use of two-step temperature growth, that is, the growth at high temperatures such as $420^{\circ} \mathrm{C}$ on a $350^{\circ} \mathrm{C}$ pre-grown layer. We also used the insertion of various buffer layers between the substrate and the active InSb layer, such as AlSb, GaAs and a $\mathrm{GaSb} / \mathrm{AlSb}$ superlattice, to reduce the lattice mismatch. The best results included the GaSb/AlSb

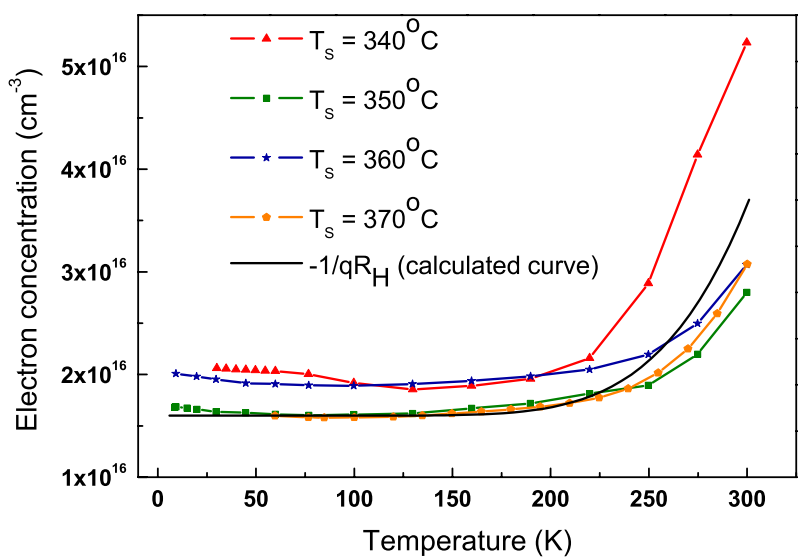

Fig. 2. Experimental carrier concentration as a function of measured temperature for InSb/GaAs.

superlattice. Two XRD patterns of InSb/Si films grown at 380 and $420^{\circ} \mathrm{C}$ with a $\mathrm{GaSb} / \mathrm{AlSb}$ superlattice are presented in Fig. 1b. The strain between the layers resulted in the right shoulder of the InSb peak. The InSb peaks related to the (004) plane appeared with quite high intensity. For the sample grown at $420^{\circ} \mathrm{C}$, the FWHM was found to be approximately 951 arcseconds. This value was smaller than those of the samples of InSb/Si grown at other conditions. The lattice constant of this InSb film determined from the XRD peak was $6.476 \mathrm{~A}$, and this value matches closely the lattice constant of the InSb bulk. These results show that a highquality InSb epilayer can be grown on a Si substrate using the $\mathrm{GaSb} / \mathrm{AlSb}$ superlattice layer.

In order to assess the electrical properties, we performed Hall measurements on Van der Pauw $^{14,15}$ samples (4 $\mathrm{mm}$ in squares) formed on the InSb layers. These measurements gave apparent carrier mobility and concentration. Figure 2 shows the experimental carrier concentration as a function of measured temperature for InSb/GaAs. All samples are $n$-type in the entire temperature range. The experimental results were then compared with the calculation of the carrier concentration for the $2 \mu \mathrm{m}$ thick InSb films. It is well known that the carrier concentration is given by:

$$
n=\frac{-1}{q R_{\mathrm{H}}},
$$

where $q$ is the electron charge and $R_{\mathrm{H}}$ is the Hall coefficient, and for a two-layer system, it should take the form

$$
R_{\mathrm{H}}=\frac{d\left(R_{\mathrm{Hb}} \sigma_{\mathrm{b}}^{2} d_{\mathrm{b}}+R_{\mathrm{Hs}} \sigma_{\mathrm{s}}^{2} d_{\mathrm{s}}\right)}{\left(\sigma_{\mathrm{b}} d_{\mathrm{b}}+\sigma_{\mathrm{s}} d_{\mathrm{s}}\right)^{2}}
$$

where the subscripts $b$ and $s$ refer to the bulk and surface, respectively; $\sigma$ is conductivity and $d=$ $d_{\mathrm{s}}+d_{\mathrm{b}}$ is total thickness. ${ }^{17}$ For a bulk semiconductor with simultaneous electron and hole conduction 
the Hall constant $R_{\mathrm{Hb}}$ and conductivity $\sigma_{\mathrm{b}}$ are given by:

$R_{\mathrm{Hb}}=\frac{p \mu_{\mathrm{h}}^{2}-n \mu_{\mathrm{e}}^{2}}{q\left(p \mu_{\mathrm{h}}+n \mu_{\mathrm{e}}\right)^{2}}$ and $\sigma_{\mathrm{b}}=\sigma_{\mathrm{e}}+\sigma_{\mathrm{h}}=q\left(n \mu_{\mathrm{e}}+p \mu_{\mathrm{h}}\right)$,

where $n(p)$ is the electron (hole) concentration and $\mu_{\mathrm{e}}\left(\mu_{\mathrm{h}}\right)$ is electron (hole) mobility. ${ }^{18}$ The temperature dependence of $n$ and $p$ are given by the equation:

$n=p=4.28 \times 10^{15} T^{1.5}\left(m_{\mathrm{e}} m_{\mathrm{h}} / m^{2}\right)^{3 / 4} \exp \left(-E_{\mathrm{g}} / 2 k T\right)$,

where $m$ is the mass of the electron in free space and $m_{\mathrm{e}}\left(m_{\mathrm{h}}\right)$ is the effective mass of the electron (hole). ${ }^{19}$ For InSb, $m_{\mathrm{e}}=0.014 \mathrm{~m}$ and $m_{h}=0.43 \mathrm{~m}$. The band gap, $E_{\mathrm{g}}$, is taken to vary with the temperature according to

$$
E_{\mathrm{g}}=0.24-\frac{6 \times 10^{-4} T^{2}}{T+500} .
$$

First, we assume that the surface layer consists of electrons and that the surface layer is much thinner than the bulk layer. Therefore, the following identities are exposed:

$$
d=d_{\mathrm{b}}, R_{\mathrm{Hs}}=\frac{-1}{q n_{\mathrm{s}}}, \quad \sigma_{\mathrm{s}}=q n_{\mathrm{s}} \mu_{\mathrm{s}}, \quad d_{\mathrm{s}} n_{\mathrm{s}}=N_{\mathrm{s}},
$$

where $\mu_{\mathrm{s}}\left(n_{\mathrm{s}}\right)$ is electron mobility (concentration) of the surface layer and $N_{\mathrm{s}}$ is the sheet electron concentration. Second, $N_{\mathrm{s}}$ is assumed to be temperature independent and to have a value of $3.6 \times 10^{12} \mathrm{~cm}^{-2}$. Third, we assume that

$$
\frac{\mu_{\mathrm{e}}}{\mu_{\mathrm{h}}}=\frac{\mu_{\mathrm{s}}}{\mu_{\mathrm{h}}}=\frac{m_{\mathrm{h}}}{m_{\mathrm{e}}}=\frac{0.43}{0.014}=30 .
$$

We can then write the Hall constant in the form:

$$
\begin{gathered}
R_{\mathrm{H}}=\frac{d}{q} \frac{d\left[p-n\left(\frac{\mu_{\mathrm{e}}}{\mu_{\mathrm{h}}}\right)^{2}\right]-N_{\mathrm{s}}\left(\frac{\mu_{\mathrm{s}}}{\mu_{\mathrm{h}}}\right)^{2}}{\left\{d\left[p+n\left(\frac{\mu_{\mathrm{e}}}{\mu_{\mathrm{h}}}\right)\right]+N_{\mathrm{s}}\left(\frac{\mu_{\mathrm{s}}}{\mu_{\mathrm{h}}}\right)\right\}^{2}} \\
R_{\mathrm{H}}=-1.25 \times 10^{15} \frac{1798 \times 10^{-4} n+32.4 \times 10^{14}}{\left(62 \times 10^{-4} n+10.8 \times 10^{13}\right)^{2}} .
\end{gathered}
$$

If we insert the form of $n$, we have the temperature dependence of the Hall constant, which yields the black curve in Fig. 2. The experimental curves for samples grown at $340^{\circ} \mathrm{C}, 350^{\circ} \mathrm{C}, 360^{\circ} \mathrm{C}$ and $370^{\circ} \mathrm{C}$ are also shown for comparison in Fig. 2.

It can be seen that the experimental curves generally fit the form of the expression of the inverse Hall constant. The presence of growth-related defects, however, results in a somewhat different temperature dependence that depends on the characteristics of the material. Below $200^{\circ} \mathrm{C}$, the carrier concentration is quite constant, indicating that there are no more intrinsic carriers. Hence, these carriers are due to the ionized impurities (scatterers). Above $200^{\circ} \mathrm{C}$, the carrier concentration increases gradually. Because the electrons are much lighter than holes, there is a finite possibility that electrons can reach the conduction band and contribute to electrical conduction ( $n$-type) in the Hall measurements and will give the sign of the Hall constant at ordinary temperatures. For the InSb layer grown at $350^{\circ} \mathrm{C}$, the electron concentration is lowest.

The Hall mobilities measured as a function of temperature for InSb films grown on $\mathrm{GaAs}$ and $\mathrm{Si}$ substrates at different temperatures are shown in Fig. 3. From the figure, it can be seen that the temperature dependencies of the electron mobility in all samples are almost identical. The highest Hall mobility measured at room temperature as $41,100 \mathrm{~cm}^{2} / \mathrm{V}$ s can be observed in the sample grown on $\mathrm{GaAs}$ at $350^{\circ} \mathrm{C}$. It is evident that the electron mobilities of InSb layers grown on GaAs are significantly higher than those of the films grown on $\mathrm{Si}$ and that the electron mobility decreased with measurement temperature in the samples grown on $\mathrm{GaAs}$, as well as in the case of InSb on Si. According to Matthiessen's rule, the electron mobility of the InSb layer, $\mu$, can be related to $\mu_{\mathrm{b}}$ and $\mu_{\mathrm{d}}$, as follows:

$$
\frac{1}{\mu}=\frac{1}{\mu_{\mathrm{b}}}+\frac{1}{\mu_{\mathrm{d}}}
$$

where $\mu_{\mathrm{b}}$ is the intrinsic electron mobility of the bulk material and $\mu_{\mathrm{d}}$ is the mobility limited by dislocation scattering. Electrons can be scattered by the depletion potential around the dislocations and the lattice dilatation associated with the dislocations. Using the Pödör and Dexter-Seitz models, ${ }^{20,21}$ Weng et al. ${ }^{1}$ found that the dominant factor limiting the electron mobility was the lattice dilatation scattering, which is apparently consistent with the predictions of the Dexter-Seitz model. According to this model, the mobility limited by deformation potential scattering $\mu_{\mathrm{d}}$ is inversely proportional to the edge dislocation density $D$. These results, therefore, show a quantitative correlation between increasing dislocation density and decreasing electron mobility. The dislocation scattering increases as the temperature decreases, because the screen effect of free carriers decreases. Therefore, the degradation of electron mobility is observed at low temperature. Figure $3 \mathrm{a}$ shows the temperature dependence of the measured Hall mobilities for the $\mathrm{InSb} / \mathrm{GaAs}$ samples. We can see that the electron mobility for the sample grown at $360^{\circ} \mathrm{C}$ is $30,800 \mathrm{~cm}^{2} / \mathrm{V} \mathrm{s}$ and $19,500 \mathrm{~cm}^{2} / \mathrm{V} \mathrm{s}$ at room temperature (RT) and $77 \mathrm{~K}$, respectively. However, for the sample grown at $340^{\circ} \mathrm{C}$, the room temperature 

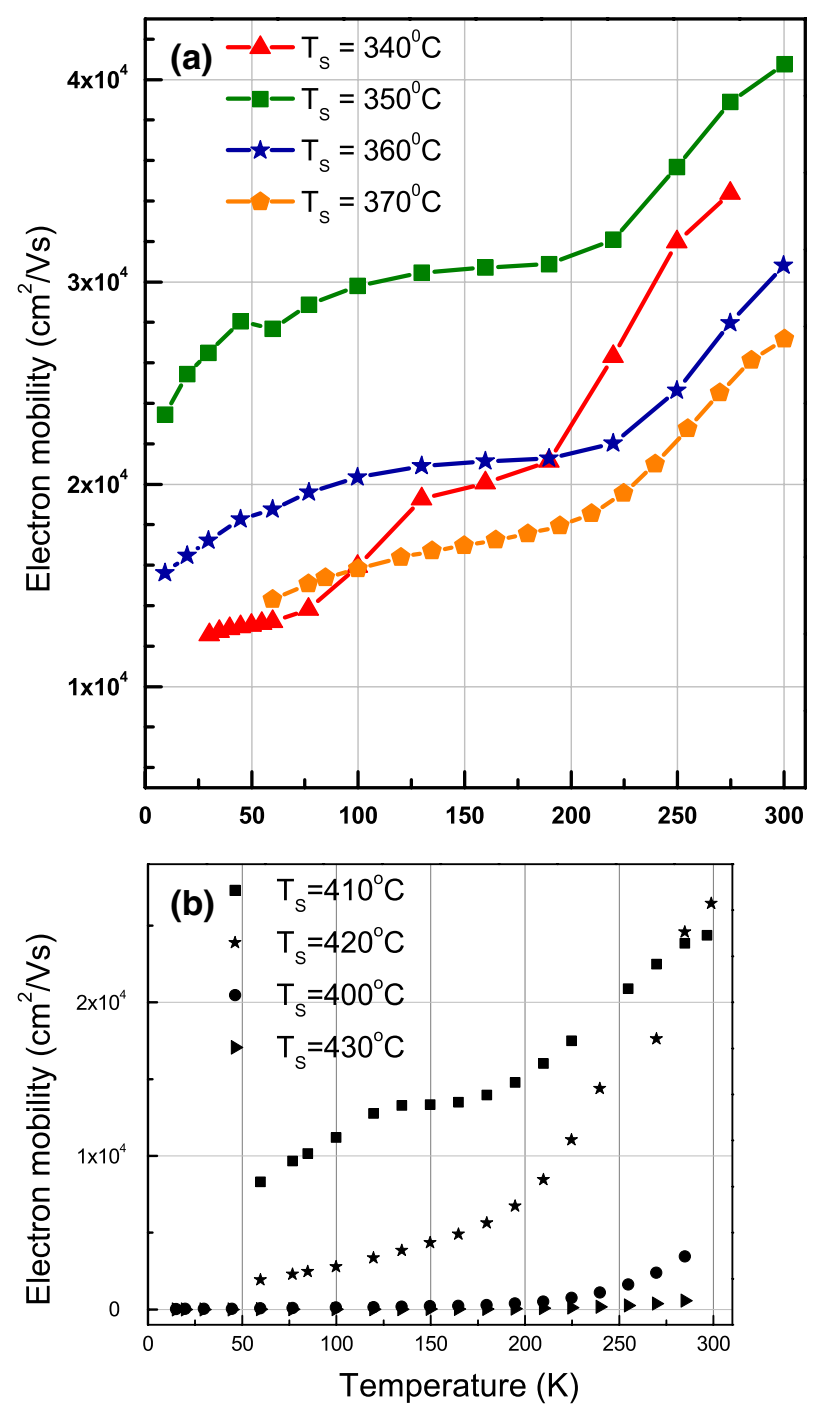

Fig. 3. Temperature-dependent electron mobilities of the $\operatorname{lnSb}$ layers (a) on GaAs and (b) on $\mathrm{Si}$.

mobility was found to be higher, but it changed significantly when the measurement temperature decreased. We obtained mobilities of $32,000 \mathrm{~cm}^{2} / \mathrm{V} \mathrm{s}$ and $13,800 \mathrm{~cm}^{2} / \mathrm{V} \mathrm{s}$ at $300 \mathrm{~K}$ and at $77 \mathrm{~K}$ (reduced by $57 \%$ compared to $36 \%$ for sample grown at $360^{\circ} \mathrm{C}$ ), indicating that more defects had formed due to unsuitable growth temperature. For the sample grown at $350^{\circ} \mathrm{C}$, the RT electron mobility improved to $41,000 \mathrm{~cm}^{2} / \mathrm{V} \mathrm{s}$, corresponding to $2.9 \times 10^{16} \mathrm{~cm}^{-3}$ of carrier concentration. The $30 \%$ reduction in electron mobility as the temperature decreased was smallest, suggesting the good quality of the film. This highest mobility was higher than the mobilities reported for $2 \mu \mathrm{m}$ MBE InSb grown directly on GaAs samples done by other groups. ${ }^{1,22}$

The samples grown at $350^{\circ} \mathrm{C}$ and $370^{\circ} \mathrm{C}$ showed the same carrier concentration at low temperatures (Fig. 2, green and orange curves) - thus the same number of ionized impurities-but markedly different mobilities. Apparently, this mobility difference cannot be an effect of carrier concentration or ionized impurity concentration. Thus, it is due to dislocation scattering with different dislocation densities and is not due to ionized impurity scattering. Generally, the mobility due to ionized impurity scattering scales with the concentration of ionized impurities. The low temperature carrier concentrations in our samples were $1.6 \times 10^{16} \mathrm{~cm}^{-3}$ $\left(350^{\circ} \mathrm{C}\right.$ and $370^{\circ} \mathrm{C}$ sample) and $2 \times 10^{16} \mathrm{~cm}^{-3}\left(340^{\circ} \mathrm{C}\right.$ and $360^{\circ} \mathrm{C}$ sample), which would correspond to a change of mobility due to ionized impurity scattering by a maximum of $25 \%$. Clearly, the low temperature mobility (low temperature chosen to exclude the influence of intrinsic carriers on mobility) of the $350^{\circ} \mathrm{C}$ sample was larger, far above $25 \%$, than the mobilities of the $340^{\circ} \mathrm{C}$ and $360^{\circ} \mathrm{C}$ samples (the samples with the higher carrier concentration at low temperature). The higher mobility of the $350^{\circ} \mathrm{C}$ sample can thus not be explained by ionized impurity scattering only-clearly there has to be an increase in mobility due to reduced dislocation scattering.

By comparing Fig. 3a and b, one observes that $\mathrm{InSb} / \mathrm{Si}$ exhibits much lower room temperature mobilities and faster degradation at low temperatures than does InSb/GaAs, because the increase or decrease in the electron mobilities corresponds to a decrease or increase in threading dislocation densities, respectively. Therefore, this strongly suggests that dislocation scattering plays an important role in degradation of the electron mobilities of the InSb films. In the case of InSb/Si, more dislocations can be generated in the grown InSb layer than those in the case of InSb/GaAs. The highest RT mobility was found to be $26,400 \mathrm{~cm}^{2} / \mathrm{V}$ s for the sample grown at $420^{\circ} \mathrm{C}$, corresponding to $n=2.5 \times 10^{16} \mathrm{~cm}^{-3}$, which is similar to the best samples on GaAs.

Finally, we describe some results of the growth and investigation of diluted magnetic semiconductor (In,Mn)Sb films with a thickness of $1 \mu \mathrm{m}$. The samples were investigated in respect of their magnetotransport and magnetization properties. The samples were $p$ type, with a hole concentration of $1.75 \times 10^{19} \mathrm{~cm}^{-3}$ and a mobility of $176 \mathrm{~cm}^{2} / \mathrm{V} \mathrm{s}$ at $300 \mathrm{~K}$. Figure 4 shows the magnetoresistance $R_{\text {sheet }}$ and Hall resistance $R_{\text {Hall }}$ at $5 \mathrm{~K}$, when the magnetic field was applied parallel to the layer plane. As shown in Fig. 4a, a small initial positive magnetoresistance region followed by a negative magnetoresistance at high fields was observed. A similar behavior has also been observed in ferromagnetic (Ga,Mn)Sb random alloys. ${ }^{23}$ This feature suggested the presence of ferromagnetic ordering at low temperature in the samples. The change in negative magnetoresistance, however, was slight. A study of spin scattering interactions between the localized magnetic moments of the substitutional Mn acceptors and the holes in an impurity band may help us obtain a better understanding of this behavior. We also performed Hall measurement at $5 \mathrm{~K}$, with the magnetic field applied perpendicularly to the layer plane. It is well known that Hall resistance in a 

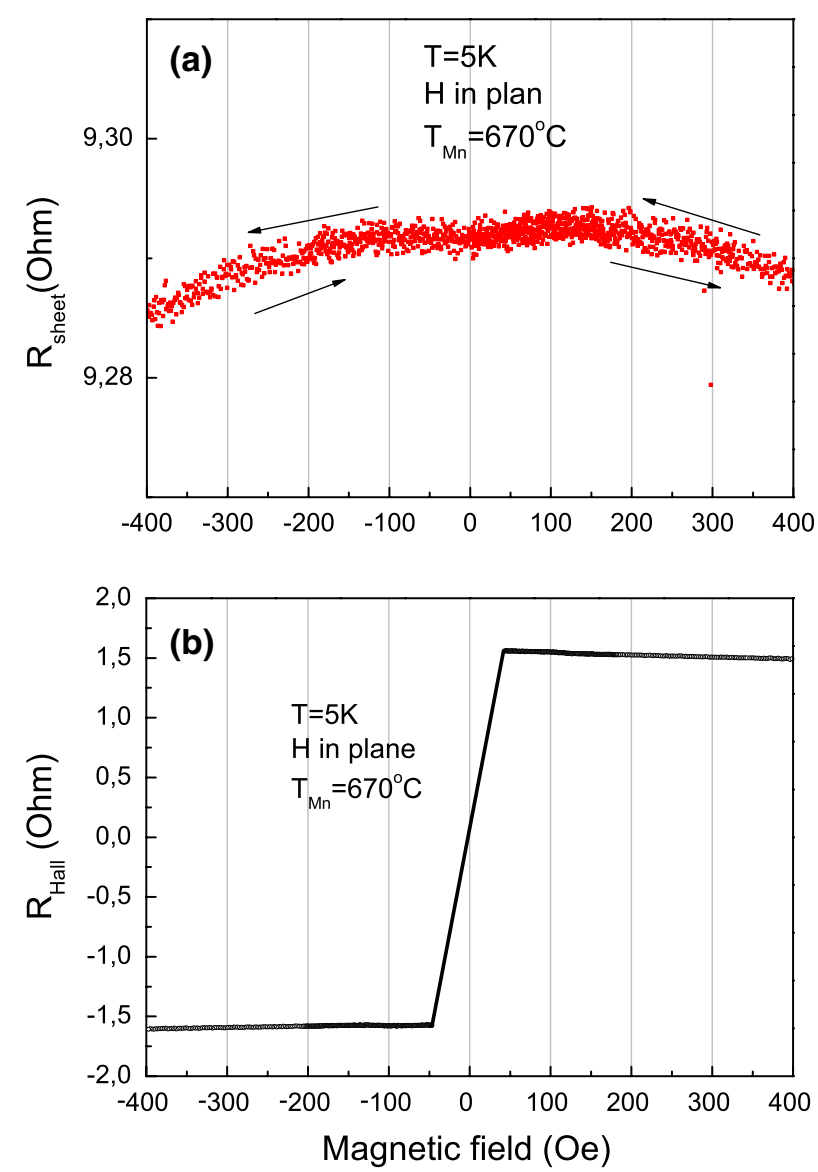

Fig. 4. Magnetic field dependence of (a) sheet resistance and (b) Hall resistance at $5 \mathrm{~K}$ of $(\mathrm{In}, \mathrm{Mn}) \mathrm{Sb}$ grown at $T_{\mathrm{Mn}}=670^{\circ} \mathrm{C}$.

ferromagnetic material can be expressed by the following equation: $R_{\text {Hall }}=\left(R_{\mathrm{O}} / d\right) B+\left(R_{\mathrm{A}} / d\right) M$, where $R_{\mathrm{O}}$ and $R_{\mathrm{A}}$ are ordinary and anomalous Hall coefficients, respectively, $B$ is the applied magnetic field, $M$ is the magnetization, and $d$ is the sample thickness. The second term represents the anomalous Hall effect, which is properly caused by anisotropic scattering between the carriers and local magnetic moments of $\mathrm{Mn}$ atoms. Since, in our sample, the carrier concentration was quite high, the first term can be negligible when compared with the second term. The Hall resistance, $R_{\text {Hall }}$, therefore, is proportional to the perpendicular component of the magnetization. As shown in Fig. 4b, the $R_{\text {Hall }}(B)$ curve shows a characteristic overshoot. It is evidently related to the small positive magnetoresistance in the same field range. The rapid increase and saturation at low fields of $R_{\text {Hall }}$ gradually become apparent when the temperature is $5 \mathrm{~K}$. The data may then be compared with the plot in Fig. 5a of the magnetization measured at $5 \mathrm{~K}$, when the magnetic field was applied perpendicularly to the plane. One observes that the two curves do not completely agree with each other. The $M(B)$ curve exhibits a clear square hysteresis loop, and the magnetization is seen to saturate at higher fields,
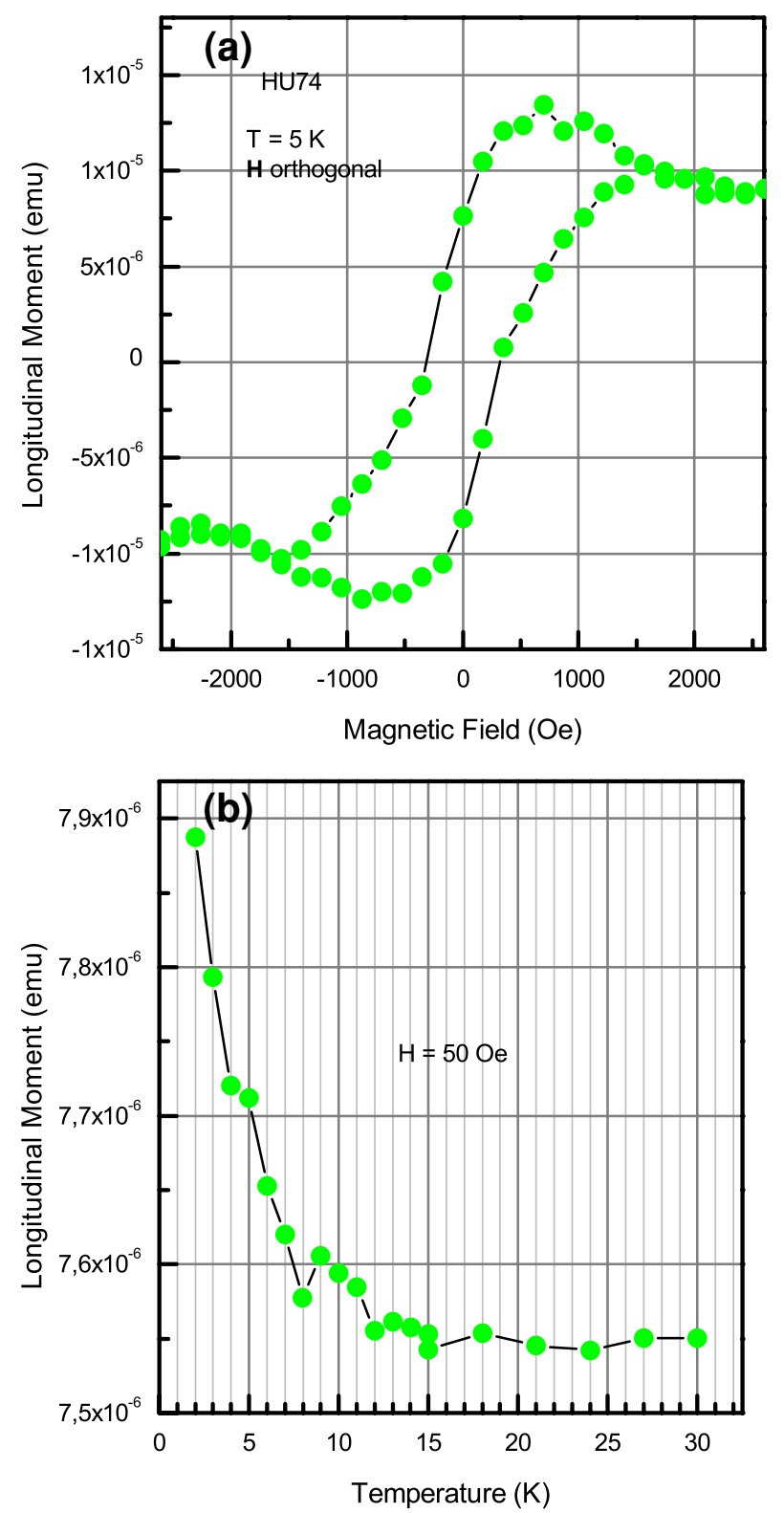

Fig. 5. (a) Magnetic field-dependent magnetization at $5 \mathrm{~K}$ when the field was applied perpendicularly to the layer plane, and (b) temperature-dependent magnetization with a perpendicular field of $50 \mathrm{Oe}$ in InMnSb/GaAs.

notably different from the anomalous Hall effect. That the easy axis of magnetization in this InMnSb/ GaAs sample is perpendicular to the layer plane, may explain the difference. The in-plane applied magnetic field in Hall measurements, in contrast to the magnetization experiments, could result in some new effects due to electron scattering. To consider electron scattering in our study, however, we need a better understanding of the physics behind the process. Figure $5 \mathrm{~b}$ shows the temperature-dependent magnetization results of the sample in a perpendicular field of 50 Oe. From this plot, a Curie temperature below $15 \mathrm{~K}$ was estimated. 


\section{CONCLUSION}

To conclude, we have described the growth of high-quality InSb films on (001) GaAs as well as (001) Si substrates using molecular-beam epitaxy. The highest RT electron mobility was 41,000 $\mathrm{cm}^{2} / \mathrm{V}$ s for a $2 \mu \mathrm{m}$-thick InSb film on GaAs grown at a substrate temperature of $350^{\circ} \mathrm{C}$. Our results indicate a significant correlation between the crystal quality of InSb films and the growth condition such as substrate temperature, growth rate, $\mathrm{Sb} / \mathrm{In}$ flux ratio, and the substrate tilting. Our results of (In,Mn)Sb films on GaAs grown at the same conditions as the best InSb/GaAs sample showed the presence of ferromagnetic ordering at low temperatures and a Curie temperature below $15 \mathrm{~K}$.

There remain a number of open questions concerning the transport and magnetic properties of (In, $\mathrm{Mn}) \mathrm{Sb}$ as functions of Mn concentration. Since (In,Mn)Sb is the III-Mn-V semiconductor with the largest lattice constant, and some of its band parameters differ considerably from those of the other III-Mn-V semiconductors, investigation of its properties provides a unique case for further evaluation of the ferromagnetic semiconductor theories.

\section{ACKNOWLEDGEMENT}

The authors would like to thank O. Bierwagen and M.P. Semtsiv at Humboldt University in Berlin, and J. Herfort at the Paul Drude Institute in Berlin for helpful discussions and assistance.

\section{REFERENCES}

1. X. Weng, N.G. Rudawski, P.T. Wang, and R.S. Goldman, J. Appl. Phys. 97, 043713 (2005).

2. E. Michel, G. Singh, S. Slivken, P. Bove, I. Ferguson, and M. Razeghi, Appl. Phys. Lett. 65, 3338 (1994).
3. V.K. Dixit, B. Bansal, V. Venkataraman, H.L. Bhat, G.N. Subbanna, K.S. Chandrasekharan, and B.M. Arora, Appl. Phys. Lett. 80, 2102 (2002).

4. D.L. Partin, L. Green, and J. Heremans, J. Electron. Mater. 23, 75 (1994).

5. J.-I. Chyi, D. Biswas, S.V. Iyer, N.S. Kumar, H. Morkoc, R. Bean, K. Zanio, H.-Y. Lee, and H. Chen, Appl. Phys. Lett. 54, 1016 (1989).

6. S.V. Ivanov, A.A. Boudza, R.N. Kutt, N.N. Ledentsov, B.Ya. Meltser, S.S. Ruvimov, S.V. Shaposhnikov, and P.S. Kop'ev, J. Cryst. Growth. 156, 191 (1995).

7. R. Fischer, H. Morkoç, D.A. Neumann, H. Zabel, C. Choi, N. Otsuka, M. Longerbone, and L.P. Erickson, J. Appl. Phys. 60, 5 (1986).

8. H.C. Lu, H.R. Fetterman, C.J. Chen, C. Hsu, and T.M. Chen, Solid-State Electron. 36, 533 (1993).

9. D.M. Li, M. Yamazaki, T. Okamoto, T. Tambo, and C. Tatsuyama, Appl. Surf. Sci. 130-132, 101 (1998).

10. W.K. Liu, J. Winesett, and W. Ma, J. Appl. Phys. 84, 4 (1997).

11. M. Mori, N. Akae, K. Uotani, N. Fujimoto, T. Tambo, and C. Tatsuyama, Appl. Surf. Sci. 216, 569 (2003).

12. S.F. Fang, K. Adomi, S. Iyer, H. Morkoç, H. Zabel, C. Choi, and N. Otsuka, J. Appl. Phys. 68, 7 (1990).

13. Bede Scientific QC1a Diffractometer, User guide, England (1997).

14. L.T. van der Pauw, Philips Tech. Rev. 20, 220 (1958).

15. O. Bierwagen, R. Pomraenke, S. Eilers, and W.T. Masselink, Phys. Rev. 70, 165307 (2004).

16. X. Weng, R.S. Goldman, D.L. Partin, and J.P. Heremans, J. Appl. Phys. 88, 11 (2000).

17. R.L. Petritz, Phys. Rev. 110, 1254 (1958).

18. J.S. Blakemore, Solid State Physics (Cambridge University Press, 1985), pp. 330-372.

19. R.A. Smith, Semiconductor, 2nd edn (Cambridge University Press, 1978), pp. 77-86.

20. B. Pödör, Phys. Status Solidi 16, K167 (1966).

21. D.L. Dexter and F. Seitz, Phys. Rev. 86, 964 (1952).

22. P. Songpongs, T.G. Andersson, M.J. Ekenstedt, J.R. Söderström, and M.M. Cumming, Appl. Phys. Lett. 65, 1433 (1994).

23. H. Luo, B.D. McCombe, M.H. Na, K. Mooney, F. Lehmann, X. Chen, M. Cheon, S.M. Wang, Y. Sasaki, X. Liu, and J.K. Furdyna, Physica E 12, 330 (2002). 